\title{
Potencial alelopático de lixiviados de cultivos de cobertura y malezas sobre plantas jóvenes de vid
}

Uliarte, E.M.; C.A. Parera, R.F. del Monte, E.E. Alessandria y A.D. Dalmasso

\begin{abstract}
RESUMEN
Las interacciones entre la vid y especies herbáceas pueden involucrar competencia por recursos y alelopatía. Se presume que determinados cultivos de cobertura y malezas poseen efectos alelopáticos sobre el crecimiento de las cepas. Los objetivos del trabajo fueron: a) evaluar el efecto de los lixiviados de riego de diversas coberturas vegetales sobre plantas de vid y b) determinar su efecto inhibitorio sobre la germinación de semillas. Se efectuaron experimentaciones de campo e invernadero, con cultivos de cobertura y vides en macetas. Se utilizaron 11 lixiviados de diferentes cubiertas vegetales y un testigo con suelo descubierto, con un diseño completamente al azar y cinco repeticiones. Se evaluó expresión vegetativa y productiva de las vides. Se realizaron pruebas de germinación sobre semillas, utilizando lixiviados del riego y diferentes concentraciones de la solución acuosa. No se detectó efecto negativo en el crecimiento vegetativo ni en la producción de uva en plantas jóvenes de vid, ni tampoco en la germinación de otras especies vegetales. El ensayo en macetas evidenció que existe una interacción entre las coberturas y el suelo, mediante la cual el balance de nutrientes es alterado según la especie, y puede afectar positiva o negativamente el crecimiento de las vides.
\end{abstract}

Palabras clave: Vitis vinífera, coberturas vegetales, interacción, alelopatía.

Uliarte, E.M.; C.A. Parera, R.F. del Monte, E.E. Alessandria and A.D. Dalmasso 2016. Allelopathic potential of leachates of cover crops and weeds on young vines. Agriscientia 33 (2): 99-111

\section{SUMMARY}

Interactions between vines and herbaceous species may involve competition for resources and allelopathy. It is presumed that certain cover crops and weeds have allelopathic effects on the growth of grapevines. The objectives were: a) to evaluate the effect of leachate from irrigation of different cover crops on vines 
and b) to determine its inhibitory effect on seed germination. Greenhouse and field experiments were conducted with cover crops and potted vines. Eleven leachates from different green covers and a bare soil control were tested with a randomized design and five replicates. The vegetative and productive expression of the vines was evaluated. Seed germination tests were conducted using leachate from irrigation and different concentrations of the aqueous solution. Adverse effects were not detected in the vegetative growth, the grape production in young vines or the germination of other plant species. The pot trial showed that there is an interaction between cover crops and soil, whereby the balance of nutrients is altered depending on the species. This balance can positively or negatively affect the growth of the vines.

Key words: Vitis vinífera, cover crops, interaction, allelopathy.

E.M. Uliarte y R.F. del Monte: Instituto Nacional de Tecnología Agropecuaria (INTA), E.E.A Mendoza. C.A. Parera: INTA, Centro Regional Mendoza-San Juan. E.E. Alessandria: Universidad Nacional de Córdoba, Facultad de Ciencias Agropecuarias. A.D. Dalmasso: Instituto Argentino de Investigaciones de las Zonas Áridas (IADIZA), CCT CONICET Mendoza. Correpondencia a: uliarte.ernesto@inta.gob.ar

\section{INTRODUCCIÓN}

Los cultivos de cobertura son propuestos para el manejo racional de los viñedos debido a sus múltiples beneficios asociados (Ingels et al., 1998; Groupe Columa Vigne, 2004). Sin embargo, la cobertura verde genera un consumo adicional de agua (Monteiro \& Lopes, 2007), por lo que bajo determinadas condiciones agroecológicas, por ejemplo en zonas áridas bajo riego localizado, se sugiere la utilización de especies nativas en contraposición al manejo de especies exóticas cultivadas o malezas (Uliarte et al., 2014).

Las interacciones entre la vid y los cultivos de cobertura pueden involucrar, además de competencia por recursos (Celette et al., 2009), manifestaciones alelopáticas que afecten el normal crecimiento de las cepas (Anaya, 1999; Inderjit \& Keating, 1999). Se entiende a la alelopatía como el fenómeno inhibitorio de una especie sobre otra, debido a la liberación de sustancias fitotóxicas (Lambers et al., 1998). Algunos estudios sugieren que, en viñedos manejados con una cobertura vegetal, la combinación entre competencia y alelopatía reduce el crecimiento de las plantas de vid (Bordelon \& Weller, 1997; Celette et al., 2005). Estas sustancias (aleloquímicos) pueden ser liberadas al ambiente por volatilización o lixiviado de partes aéreas de la planta, exudados de las raíces, lixiviación de los residuos de las plantas o por descomposición de la materia orgánica (Chick \& Kielbaso, 1998; Anaya, 1999).
La mayoría de los aleloquímicos pueden clasificarse como metabolitos secundarios y están relacionados con mecanismos de defensa de la planta. Entre ellos los grupos más importantes son: terpenoides, compuestos aromáticos, alcaloides y aminas, glicósidos cianogenéticos y glucosinolatos (Leicach, 2006). Existen también ciertos metabolitos primarios de significativa importancia en alelopatía como, por ejemplo, algunos ácidos y alcoholes orgánicos simples, aldehídos y cetonas, ácidos grasos y ésteres (Inderjit \& Keating, 1999). Blum (1996), basándose en diversos estudios, sugiere que la actividad alelopática se produce, no por la acción de una única sustancia, sino debido a la combinación de diferentes aleloquímicos y otros compuestos orgánicos.

Diversas malezas y especies utilizadas como coberturas verdes son citadas por su potencial efecto alelopático. Inderjit \& Keating (1999) mencionan más de cien malezas comunes en agroecosistemas con potencial actividad alelopática. Entre ellas se citan especies como Avena fatua, Bidens pilosa, Chenopodium album, Cynodon dactylon, Cyperus rotundus, Echinochloa crus-galli, Kochia scoparia, Lolium multiflorum, Portulaca oleracea, Salsola kali, Setaria spp., Sorghum halepense, Tagetes patula y T. minuta, entre otras. Entre los cultivos de cobertura indican por ejemplo a Avena sativa, Brassica spp., Festuca arundinacea, Hordeum vulgare, Medicago sativa, Secale cereale, Sorghum sudanense, Trifolium repens y pratense, Triticum aestivum y Vicia villosa. No existen antecedentes sobre el potencial efecto alelopático de las 
especies herbáceas nativas de Mendoza; sólo es posible citar algunas especies emparentadas por su género. Este es el caso de Digitaria sanguinalis, Leptochloa filiformis, Setaria faberi, S. glauca y S. viridis (Inderjit \& Keating, 1999).

Dentro de la gran diversidad de plantas, malezas y cultivadas, con potencial alelopático citadas en la literatura y la enorme cantidad de sustancias aleloquímicas involucradas en las interacciones (Leicach, 2006; Leicach, 2009), entre las especies estudiadas se ha verificado la intervención de más de una sustancia. En el caso del género Sorghum se reconoce una quinona específica llamada sorgoleona, pero se citan, además, glicósidos cianogénicos (dhurrina), los ácidos clorogénico, p-cumárico, p-hidroxibenzaldehído, gálico, ferúlico, siríngico, protocateico, benzoico, hidroxibenzoico y vainílico (Bengoa, 1983; Bais et al., 2006; Cheema et al., 2007; Sampietro, 2007). En C. dactylon se han reportado los ácidos cafeicos, clorogénico, cinámico, p-cumárico y ferúlico (Velu \& Rajagopal, 1996). Para S. cereale se mencionan: DIBOA (2,4-dihidroxi-1,4-(2H) benzoxazina-3-ona), DIMBOA (2,4-dihidroxi-7-metoxi-1,4-benzoaxin-3ona), ácidos fenólicos e hidroxámicos (Bertin et al., 2003; Macías et al., 2007). En F. arundinacea se han identificado los ácidos láctico, succínico, málico, cítrico, shikímico, glicérico, fumárico y quínico (Peters \& Luu, 1985). Los Trifolium spp. liberan compuestos fenólicos (Ohno \& Doolan, 2001; Bertin et al., 2003), mientras que para los residuos de cultivos en general, se citan los siguientes aleloquímicos: ácidos cafeico, t-cinámico, clorogénico, p-cumárico, ferúlico, p-hidroxibenzaldehído, vainíllico y siríngico (Bengoa, 1983; Sampietro, 2007).

Cabe destacar que el potencial alelopático de la mayoría de las especies citadas ha sido comprobado en bioensayos, usando lixiviados o extractos, y no necesariamente demuestran fenómenos alelopáticos de manera concluyente (Inderjit \& Keating, 1999). Dentro de un ecosistema, los fenómenos alelopáticos coexisten con la competencia por recursos (luz, agua y nutrientes) (Inderjit \& Weston, 2000) y son fuertemente influenciados por factores medioambientales de estrés en el cultivo, tales como el ataque de plagas y enfermedades, temperaturas extremas, deficiencias nutricionales, restricción hídrica, radiación solar y la acción de herbicidas (Einhellig, 1996). Estas condiciones de estrés generalmente estimulan la producción de compuestos alelopáticos. Se ha comprobado asimismo que los microorganismos del suelo influyen en la producción de sustancias alelopáticas (Inderjit, 2005).

Una gran parte de los estudios relacionados con la alelopatía han sido desarrollados en ambientes controlados e intentado separar los dos mecanismos de interferencia (competencia y alelopatía), lo cual ha llevado a malinterpretar el fenómeno alelopático (Inderjit \& del Moral, 1997). Diversos investigadores expresan su preocupación por la falta de previsibilidad de los bioensayos de laboratorio para explicar la interferencia aleloquímica a campo, y afirman que es necesario resaltar la importancia de los estudios de ecología y química de los suelos, tanto en el laboratorio como en el campo (Inderjit, 2001).

Se presume que los lixiviados del riego de determinados cultivos de cobertura y malezas poseen efectos alelopáticos sobre el crecimiento de la vid. Como paso previo a la difusión de especies nativas promisorias como cultivos de cobertura, en función de su mayor adaptación a futuros escenarios climáticos y elevada eficiencia en el uso del agua (Uliarte et al., 2013; Uliarte et al., 2014), es conveniente verificar que su introducción en el sistema productivo no implique la liberación de sustancias inhibitorias para el crecimiento de la planta de vid.

El objetivo del trabajo consistió en evaluar el efecto del agua de percolación proveniente del riego de diversos cultivos de cobertura, sobre plantas jóvenes de vid creciendo en macetas y corroborar su efecto inhibitorio sobre la germinación de semillas de especies sensibles o indicadoras.

\section{MATERIALES Y MÉTODOS}

\section{Experimentación de campo}

Especies bajo estudio, sitio y diseño experimental

El estudio se desarrolló en la Estación Experimental Mendoza del INTA, situada en Luján de

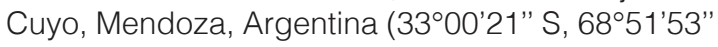
O, $929 \mathrm{msnm}$ ).

Se seleccionaron seis gramíneas nativas: Digitaria californica (Benth.) Henrard, Leptochloa dubia (Kunth) Nees, Setaria mendocina Phil., Pappophorum caespitosum R.E. Fr., Sporobolus cryptandrus (Torr.) A. Gray y Nassella tenuis (Phil.) Barkworth, como especies dominantes en tres viñedos ubicados en diferentes zonas vitícolas de Mendoza. Tres especies exóticas cultivadas: Trifolium repens L. (leguminosa), Festuca arundinacea Schreb. (gramínea), ambas perennes, y Secale cereale L. (gramínea anual invernal). Finalmente dos malezas perennes de actividad estival de amplia difusión: Sorghum halepense (L.) Pers. y Cynodon dactylon (L.) Pers.

Los datos meteorológicos del sitio de experi- 
mentación, el detalle de la metodología y el criterio utilizados para seleccionar las especies a ensayar se describen en Uliarte et al. (2014).

Se diseñó un ensayo con los cultivos de cobertura implantados en macetas a campo para separar el efecto de competencia y alelopatía con las vides. Se utilizaron macetas plásticas de 30 litros de capacidad con un sustrato compuesto por $60 \%$ de turba, $30 \%$ de perlita y $10 \%$ de tierra francoarenosa (libre de malezas).

Las especies perennes fueron establecidas en el mes de septiembre de 2007 mediante trasplante, mientras que la única anual ( $S$. cereale) fue sembrada en otoño de 2008 y 2009. Las unidades experimentales (macetas) fueron distribuidas en diseño completamente al azar con 11 tratamientos determinados por los lixiviados de diferentes cultivos de cobertura, un testigo con lixiviado de suelo descubierto y cinco repeticiones por tratamiento. Se definieron riegos con una frecuencia de 7 a 21 días y un volumen en exceso de 15 litros por maceta, utilizando agua de pozo (Tabla 1), con el objeto de recuperar un volumen de lixiviación de al menos 6 litros, para posteriormente regar las cepas jóvenes de vid trasplantadas en las macetas. Los riegos sobre las coberturas verdes se efectuaron en forma de lluvia mediante una regadera, para obtener un lixiviado de la totalidad de la parte aérea y radical de las plantas. Mayor detalle respecto del manejo de las macetas se presenta en Uliarte et al. (2014).

Tabla 1. Análisis del agua de riego, proveniente de pozo, utilizada para regar las macetas del ensayo.

\begin{tabular}{|c|c|}
\hline $\operatorname{CEA}(1)\left(\mathrm{dS} \mathrm{m}^{-1}\right)$ & 1,01 \\
\hline Sales probables $\left(\right.$ meq $\left.^{-1}\right)$ & 11,9 \\
\hline $\mathrm{pH}$ & 7,32 \\
\hline Cloruros (meq $\left.\mathrm{l}^{-1}\right)$ & 2,1 \\
\hline Carbonatos $\left(\right.$ meq l$\left.^{-1}\right)$ & 0,0 \\
\hline Bicarbonatos $\left(\mathrm{meq}^{-1}\right)$ & 2,9 \\
\hline Sulfatos $\left(\right.$ meq $\left.{ }^{-1}\right)$ & 5,7 \\
\hline Calcio + Magnesio $\left(\right.$ meq l-1 $\left.^{-1}\right)$ & 7,9 \\
\hline Calcio $($ meq l-1 $)$ & 6,4 \\
\hline Magnesio $($ meq l-1) & 1,5 \\
\hline Sodio $\left(\right.$ meq $\left.\mathrm{l}^{-1}\right)$ & 2,8 \\
\hline RAS (2) & 1,4 \\
\hline RAS ajustado & 1,9 \\
\hline
\end{tabular}

Referencias: (1) Conductividad eléctrica actual. (2) Relación de absorción de sodio.

Las muestras de los lixiviados fueron enviadas al Instituto de Biotecnología de la Universidad Nacional de San Juan, con el propósito de efectuar una aproximación química básica en la búsqueda de sustancias alelopáticas

Frente a cada maceta, que contenía el tratamiento con cobertura vegetal, se dispusieron plantas jóvenes de Vitis vinifera cv. Malbec de origen clonal (clon Cot 598), con un brote en verde de 4 a 8 hojas ( 4 meses de edad), adquiridas en un vivero de la zona y trasplantadas en el mes de noviembre de 2007 a macetas de características y sustrato idénticos al utilizado para las diferentes coberturas vegetales. Las plantas de vid fueron conducidas en un sistema de espaldero, y se les hizo poda de formación durante el invierno. Se efectuaron tratamientos fúngicos preventivos periódicos contra peronóspora y oidio, aplicando oxicloruro de cobre (Caurifix 84\% WG) y azufre (Kumulus 80\% WP). Las vides no fueron fertilizadas y durante el ensayo no presentaron síntomas de deficiencias nutricionales.

\section{Crecimiento de las plantas de vid}

Se efectuaron evaluaciones de los siguientes parámetros de expresión vegetativa y productiva de las plantas de vid:

- Cálculo del área foliar, a través del conteo del número de hojas por planta y extracción de una muestra de cuatro hojas por cada brote principal (una hoja de la base, dos del centro y una del extremo), realizado al finalizar la temporada, para determinar la superficie por hoja mediante un medidor láser de área foliar (Cl-203CA, CID Inc., Camas, WA, USA).

- Cálculo del diámetro de tronco a través de la medición de su perímetro, medido a 2,5 cm desde el cuello de la planta.

- Medición de la longitud final de brote.

- Peso de uva producida por planta.

A mediados del mes de junio de 2010, las plantas de vid en macetas regadas durante tres temporadas con agua proveniente de lixiviados de las diferentes coberturas vegetales, fueron extraídas para evaluar el peso fresco de la parte aérea (sarmientos y madera vieja) y de raíces. A continuación, el material fue llevado a estufa de secado (BHR, Dalvo, Santa Fe, Argentina) a $65^{\circ} \mathrm{C}$ durante 24 horas, para obtener el peso de materia seca.

Al finalizar el ensayo experimental se evaluaron la salinidad (conductividad eléctrica en extracto de saturación) y los niveles nutricionales (nitrógeno, fósforo y potasio totales por espectrofotometría de absorción atómica) de los sustratos pertenecientes a las macetas de las plantas de vid, para verificar si el tratamiento aplicado indujo algún cambio químico en el suelo. 


\section{Estudios en invernadero}

Complementariamente se establecieron ensayos en invernadero para verificar el potencial alelopático de los lixiviados sobre pequeñas plantas de vid provenientes de estaca. Los estudios se repitieron en dos períodos. En el primero, iniciado en enero de 2009, se utilizaron plantas del cv. Malbec procedentes de selección masal a pie franco, mientras que en la repetición del ensayo, iniciada a fines de diciembre de 2009, se emplearon estacas de 2 años del cv. Malbec (clon Cot 598) a pie franco. En ambos casos las estacas fueron trasplantadas a macetas de $750 \mathrm{~cm}^{3}$ de capacidad, con un sustrato compuesto de $70 \%$ orujo y $30 \%$ turba rubia.

Las macetas fueron dispuestas en un diseño experimental completamente aleatorizado, con los 11 tratamientos, un testigo y cinco repeticiones. Las plantas mantenidas en el invernadero fueron regadas con el agua recuperada del lixiviado de los diferentes tratamientos con cobertura vegetal, con una frecuencia de tres riegos semanales de $250 \mathrm{~cm}^{3}$ por maceta.

La utilización de estacas de vid, creciendo activamente dentro de un invernáculo, en macetas más pequeñas con una elevada proporción de raíces y una mayor frecuencia de riego, intentó favorecer la manifestación del potencial efecto inhibidor de los lixiviados sobre plantas aparentemente más sensibles.

A fines de julio de 2009 y de abril de 2010 se determinó el peso fresco de brotes y raíces. Luego el material fue llevado a estufa de secado (BHR, Dalvo, Santa Fe, Argentina) a $65^{\circ} \mathrm{C}$ durante 24 horas para obtener el peso de materia seca.

\section{Pruebas de germinación}

Se realizaron pruebas biológicas de germinación de semillas para verificar la acción de potenciales aleloquímicos presentes en los lixiviados de las coberturas vegetales.

\section{Sobre una especie sensible: Lactuca sativa}

En una primera instancia, se dispusieron 25 semillas de L. sativa cv. Rapidimor clara (poder germinativo: 92\%) por cada caja de Petri (9 cm de diámetro). El papel de filtro ubicado en las cajas fue humedecido con $5 \mathrm{ml}$ del lixiviado proveniente de las diferentes coberturas vegetales. Cinco repeticiones por tratamiento se ubicaron completamente al azar en una cámara germinadora tipo Jacobsen (GBM10, Dalvo, Santa Fe, Argentina) a $25^{\circ} \mathrm{C}$ y en oscuridad durante cinco días; al finalizar se determinaron porcentaje de germinación y longitud de hipocótilo y radícula.

Como no se detectaron efectos destacables con la dilución propia del lixiviado de un riego habitual de las macetas, se decidió efectuar su concentración y comprobar su efecto en la germinación de $L$. sativa. Previamente se efectuó un bioensayo exploratorio, utilizando una muestra compuesta por las distintas repeticiones de cada lixiviado, con el objetivo de escoger las especies más representativas. En esta instancia se seleccionaron los tratamientos de Sporobolus cryptandrus, Nassella tenuis, Sorghum halepense y suelo descubierto, utilizando como testigo al agua destilada. Los lixiviados fueron llevados a tres niveles de concentración (2,5:1, 5:1 y 10:1) obtenidos mediante el uso de un rotovapor (Büchi Waterbath B-480, Flawil, Suiza), previa extracción de las potenciales sustancias alelopáticas con éter etílico y acetato de etilo. Estos concentrados se utilizaron para realizar pruebas de germinación con la misma metodología citada previamente.

\section{Sobre otras especies}

Se concretaron pruebas de germinación utilizando semillas de dos especies comúnmente cultivadas como coberturas verdes, regadas con agua de lixiviado proveniente de todas las especies estudiadas, para verificar si podían inhibir la emergencia de otros cultivos de cobertura. Se situaron 25 semillas de vicia (Vicia sativa) y trébol blanco (Trifolium repens) en macetas de $750 \mathrm{~cm}^{3}$ conteniendo tierra de embanque (franco-arenosa) con cuatro y cinco repeticiones por tratamiento respectivamente. Se mantuvieron bajo invernáculo durante 7 días con un riego total de $500 \mathrm{ml}$ con agua de lixiviado de coberturas. Se determinaron porcentaje de germinación y longitud de hipocótilo y radícula.

\section{Análisis estadístico de los resultados}

Para el análisis estadístico de los datos se realizaron estudios de varianzas mediante el software Infostat versión 2011 (Di Rienzo et al., 2011). Los resultados obtenidos se presentan en cuadros y gráficos. Las diferencias entre las medias de los tratamientos fueron comparadas empleando la prueba de Tukey $(\rho \leq 0,05)$. Se determinaron los errores estándares para las medias de los tratamientos, los cuales aparecen en figuras y tablas. 


\section{RESULTADOS Y DISCUSIÓN}

\section{Experimentación de campo}

Los efectos de los lixiviados obtenidos de los distintos tratamientos sobre la longitud final de brotes y la producción no fueron significativos para ninguno de los parámetros estudiados, y se detectó una elevada variabilidad entre plantas a pesar de tratarse de un clon del cv. Malbec. Sin embargo, cabe señalar algunas tendencias observadas en la temporada 2009/10 (Figura 1): el tratamiento testigo mostró el mayor promedio de producción con baja longitud de brotes, mientras que con los lixiviados de la leguminosa ( $T$. repens), se presentó la mayor longitud de brotes y la menor producción. Esto puede explicarse por la fijación biológica de nitrógeno y su posterior lixiviación hacia las macetas de vid, lo cual fue comprobado en el análisis del sustrato (Paynel et al., 2001). Los tratamientos de S. cryptandrus y F. arundinacea exhibieron tendencia a inducir brotes cortos y baja producción,

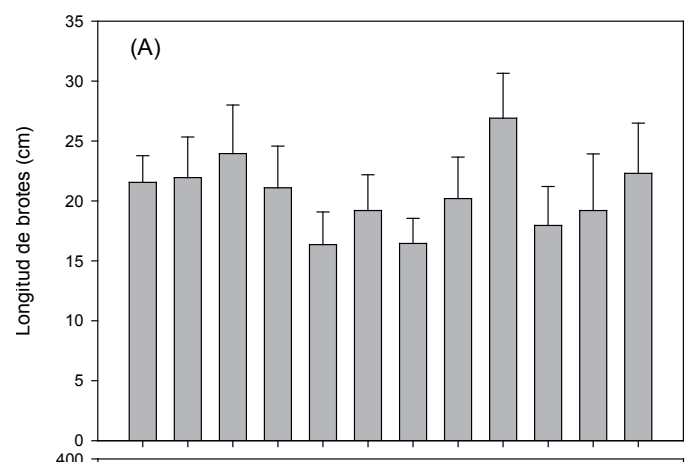

(B)

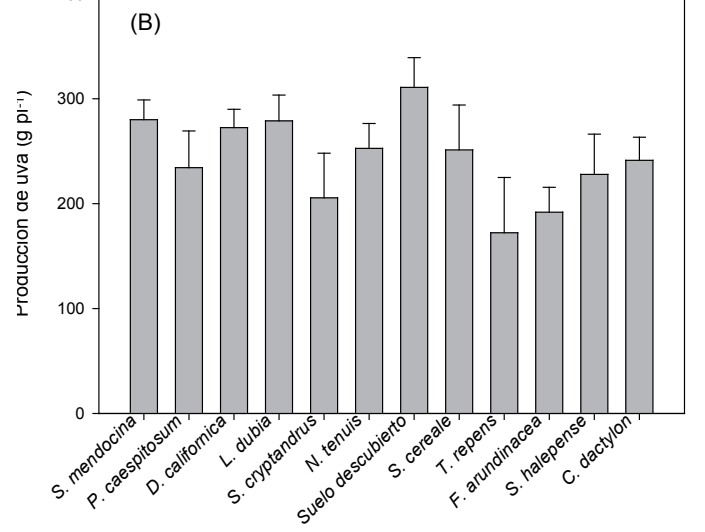

Figura 1. Longitud final de brote (A) y producción de uva (B) en plantas jóvenes de vid creciendo en macetas y regadas con el lixiviado de diferentes coberturas vegetales. Temporada 2009/10. No se presentaron diferencias significativas entre las medias (prueba de Tukey; $\rho \leq 0,05 ; n=5$ ). Las líneas verticales indican el error estándar. que puede estar relacionada con un mayor requerimiento de nutrientes, empobrecimiento del sustrato y menor concentración en el lixiviado y, por ende, hacia las plantas de vid. F. arundinacea es una especie conocida por su agresividad, y utilizada para reducir el exceso de vigor en la vid (Ingels et al., 1998). En un estudio sobre la interacción de campo entre una cobertura de $F$. arundinacea y las plantas de vid, se comprobó una disminución en el vigor de estas últimas, explicándose en parte por la competencia por agua y sugiriendo la intervención de otros mecanismos, como por ejemplo competencia por nutrientes y alelopatía (Celette et al., 2005). Bordelon \& Weller (1997) comprobaron el efecto en la reducción del crecimiento de plantas de vid en su primer año, provocado por el establecimiento previo de cultivos de cobertura, en parcelas con riego por aspersión. Aquí también se supone una combinación entre competencia y alelopatía.

Luego de tres temporadas (junio de 2010) se evaluó la producción de materia seca de madera y raíces. Estos parámetros tampoco manifestaron diferencias estadísticas significativas. De las once diferentes especies estudiadas, tres (S. cryptandrus, $S$. mendocina y $T$. repens) presentaron pesos de materia seca de raíces inferiores al testigo y sólo una ( $S$. cryptandrus) mostró menor peso de materia seca de madera (Figura 2).

En la Tabla 2 se presenta el análisis de salinidad y macronutrientes en los sustratos que contenían

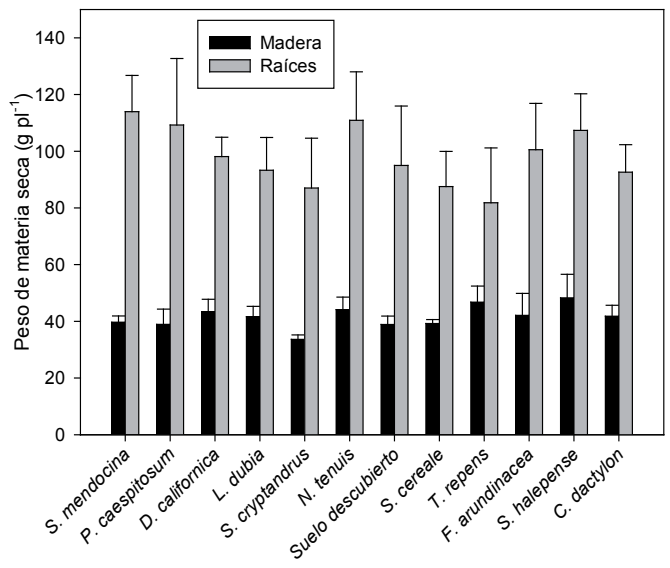

Figura 2. Producción de materia seca, discriminada en madera; vieja y del año (barras negras) y raíces (barras grises), de plantas jóvenes de vid creciendo en macetas y regadas con el lixiviado de diferentes coberturas vegetales por tres temporadas. Junio de 2010. No se presentaron diferencias significativas entre las medias (prueba de Tukey; $\rho \leq 0,05 ; n=5$ ). Las líneas verticales indican el error estándar. 
Tabla 2. Análisis final de conductividad eléctrica y macronutrientes en sustratos provenientes de plantas jóvenes de vid, creciendo en macetas y regadas por tres temporadas con el lixiviado de diferentes coberturas vegetales. Mayo de 2010 . Letras distintas indican diferencias significativas entre las medias (prueba de Tukey; $\rho \leq 0,05 ; n=5$ ), mientras que entre paréntesis se muestra el error estándar.

\begin{tabular}{|c|c|c|c|c|c|}
\hline \multicolumn{2}{|c|}{ Coberturas vegetales } & \multirow{2}{*}{$\begin{array}{c}\begin{array}{c}\text { Conductividad } \\
\text { eléctria } \\
\left.\text { (dS }^{-1}\right)\end{array} \\
259(0.32)\end{array}$} & $\begin{array}{l}\text { Nitrógeno } \\
(\%)\end{array}$ & $\begin{array}{c}\text { Fósforo } \\
\text { (\%) }\end{array}$ & $\begin{array}{c}\text { Potasio } \\
\text { (\%) }\end{array}$ \\
\hline \multirow{6}{*}{$\begin{array}{l}\text { Gramíneas } \\
\text { nativas }\end{array}$} & S. mendocina & & $0,110(0,010) b c$ & $0,110(0,004) b$ & $0,246(0,02)$ \\
\hline & P. caespitosum & $2,89(0,10)$ & $0,135(0,004) a b c$ & $0,086(0,003) \mathrm{de}$ & $0,240(0,03)$ \\
\hline & D. californica & $2,93(0,28)$ & $0,106(0,010) c$ & $0,090(0,010) \mathrm{cde}$ & $0,247(0,02)$ \\
\hline & L.dubia & $3,04(0,25)$ & $0,188(0,010) a$ & $0,094(0,003) \mathrm{bcd}$ & $0,238(0,03)$ \\
\hline & S. cryptandrus & $3,02(0,34)$ & $0,129(0,020) b c$ & $0,094(0,003) \mathrm{bcd}$ & $0,275(0,03)$ \\
\hline & N.tenuis & $2,87(0,20)$ & $0,141(0,020) a b c$ & $0,081(0,004)$ de & $0,254(0,03)$ \\
\hline Testigo & Suelo descubierto & $2,89(0,27)$ & $0,121(0,004) b c$ & $0,113(0,003) b$ & $0,307(0,02)$ \\
\hline \multirow{3}{*}{ Introducidas } & S. cereale & $2,76(0,15)$ & $0,115(0,010) b c$ & $0,071(0,003) \mathrm{e}$ & $0,267(0,02)$ \\
\hline & T. repens & $3,40(0,21)$ & $0,161(0,020) a b$ & $0,136(0,004) a$ & $0,254(0,03)$ \\
\hline & F. arundinacea & $3,28(0,38)$ & $0,101(0,010) c$ & $0,106(0,010) b c$ & $0,270(0,03)$ \\
\hline \multirow{2}{*}{ Malezas } & S. halepense & $3,29(0,24)$ & $0,122(0,010) b c$ & $0,076(0,010) \mathrm{de}$ & $0,245(0,02)$ \\
\hline & C. dactylon & $2,75(0,20)$ & $0,111(0,010) b c$ & $0,082(0,005) \mathrm{de}$ & $0,225(0,02)$ \\
\hline
\end{tabular}

plantas de vid, regadas por tres temporadas con el lixiviado de distintas especies vegetales. No se manifestaron diferencias significativas en cuanto a conductividad eléctrica ni en los contenidos de potasio en el sustrato, pero sí en los de nitrógeno y fósforo.

Todos los tratamientos regados con lixiviados de cobertura vegetal presentaron en su sustrato niveles de nitrógeno iguales o superiores a los del suelo descubierto. Los tratamientos de L. dubia y $T$. repens exhibieron los tenores más elevados distinguiéndose estadísticamente del testigo. El aumento en esta última especie se explicaría por la captación de nitrógeno atmosférico, mientras que el registrado en $L$. dubia puede atribuirse a un menor requerimiento nutricional de la especie. Sin diferenciarse significativamente, el tratamiento F. arundinacea, mostró el menor contenido de nitrógeno en el sustrato.

También se detectaron diferencias significativas en fósforo, el tratamiento con leguminosa ( $T$. repens) se distinguió del testigo y del resto de las especies por su mayor contenido de fósforo. Este efecto puede explicarse por la actividad radical (exudado de ácidos orgánicos) y una mayor actividad microbiológica del suelo, inducida por el aumento en el contenido de nitrógeno, que pueden haber promovido una mayor movilización de fósforo (Marschner et al., 1987; Whitelaw, 2000). Tres de las especies nativas ( $S$. mendocina, L. dubia y $S$. cryptandrus) y $F$. arundinacea no se diferenciaron del suelo descubierto, mientras que el resto de los tratamientos mostraron valores de fósforo significativamente menores. La reducción en el contenido de fósforo observada respecto del testigo, en la mayoría de las gramíneas, puede ser atribuida a un mayor requerimiento nutricional, que haya modificado la composición nutritiva de los lixiviados con los que posteriormente se regaron las macetas de vid. El menor contenido de este nutriente correspondió a S. cereale, la especie con mayor cantidad de plantas por maceta debido a su corto ciclo anual. La mayor cantidad de sistemas radicales en estado inicial de desarrollo, renovados por la siembra anual, pudo en este caso determinar un mayor requerimiento de fósforo (Römer \& Schilling, 1986).

\section{Experimentación en invernadero}

Como no se detectó efecto alelopático evidente de los lixiviados de las especies ensayadas, en plantas de vid creciendo en macetas (30 I) a campo, se decidió efectuar ensayos complementarios en invernadero, con macetas más pequeñas $\left(750 \mathrm{~cm}^{3}\right)$ y estacas de vid. El ensayo se repitió en 2009 y 2010 obteniendo resultados similares. Respecto a los resultados de este último año, al evaluar el área foliar total por planta y el peso de materia seca de brotes y raíces, no se manifestaron diferencias estadísticas significativas entre tratamientos (Figura 3) y nuevamente se presentó alta variabilidad entre plantas. Sin embargo, se puede destacar que el tratamiento con leguminosa ( T. repens) presentó un área foliar ligeramente mayor, posiblemente debido al citado mayor aporte de nitrógeno por la fijación biológica, mientras que $S$. cryptandrus mostró una leve reducción en el peso seco de brotes y un pequeño incremento del peso seco de raíces. En el testigo, los valores promedio 

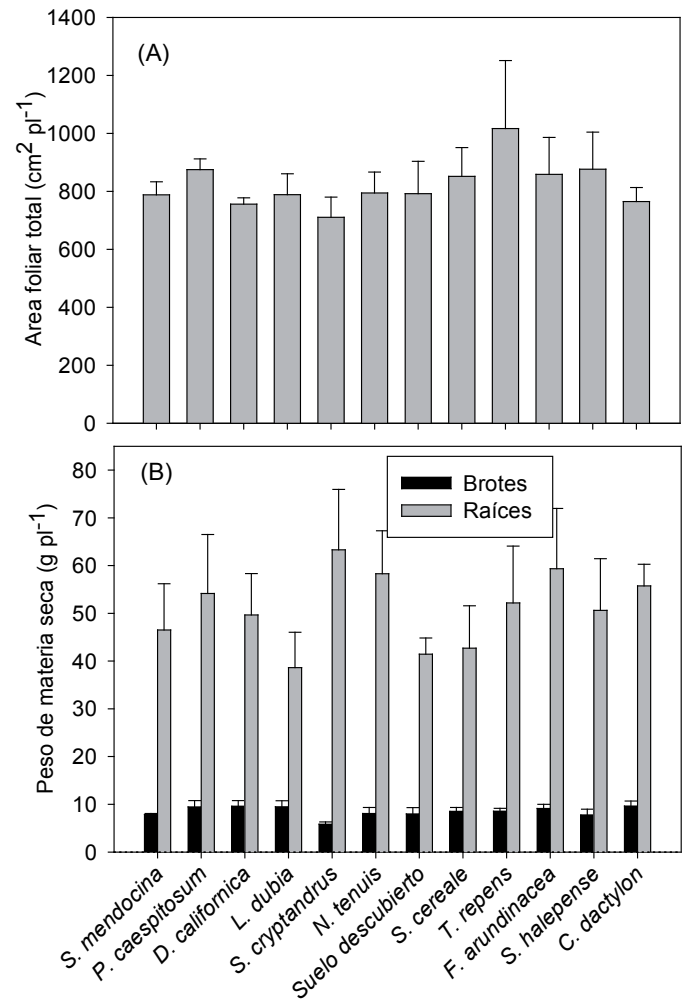

Figura 3. Área foliar (A) y producción de materia seca (B), discriminada en brotes (barras negras) y raíces (barras grises), de estacas de vid creciendo en pequeñas macetas bajo invernadero y regadas con el lixiviado de diferentes coberturas vegetales. Abril de 2010. No se presentaron diferencias significativas entre las medias (prueba de Tukey; $\rho \leq 0,05 ; n=5$ ). Las líneas verticales indican el error estándar.

del área foliar y el peso seco de brotes fueron similares a los obtenidos en los restantes tratamientos, mientras que el peso seco de raíces resultó ser uno de los más bajos (Figura 3).

Con los resultados obtenidos respecto de la producción de materia seca de las plantas de vid, luego de tres temporadas, se puede afirmar que no existió ningún efecto alelopático evidente de los lixiviados sobre el crecimiento de cepas jóvenes.

\section{Pruebas de germinación}

En la Figura 4 A se observa que ninguno de los lixiviados inhibió la germinación de L. sativa. Al evaluar la longitud de las plántulas se verificó que todos los tratamientos presentaron mayor longitud de hipocótilo respecto del testigo con agua destilada, pero no se diferenciaron del lixiviado de suelo descubierto. Los valores de $S$. cryptandrus fueron significativamente menores a los de $N$. tenuis y $S$. halepense. El tratamiento de D. californica exhibió
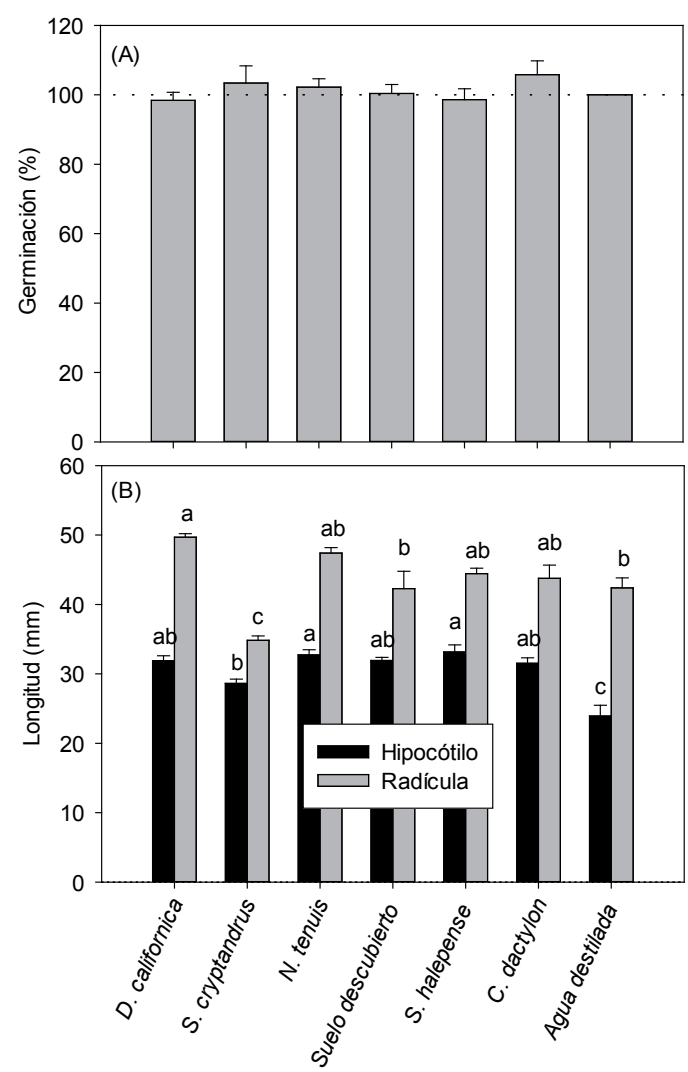

Figura 4. Efecto del lixiviado de diferentes coberturas vegetales en la germinación (A), expresada en \% respecto de agua destilada, y en la longitud de plántula (B), discriminada en hipocótilo y radícula, de Lactuca sativa cv. Rapidimor clara. Ensayo efectuado en cámara de germinación. Valores obtenidos cinco días después del tratamiento. Marzo de 2010. Letras distintas indican diferencias estadísticas significativas entre las medias (prueba de Tukey; $\rho \leq 0,05 ; n=5$ ). Las líneas verticales muestran el error estándar.

mayor longitud de radícula y el de $S$. cryptandrus mostró una longitud menor, ambos comparados con los testigos (suelo descubierto y agua destilada); el resto de las especies ensayadas no se diferenciaron de los testigos, respecto de la longitud de radícula (Figura $4 \mathrm{~B}$ ). En bioensayos de germinación adicionales efectuados durante la temporada 2009 no se detectaron diferencias significativas para germinación, longitudes de hipocótilo ni radícula (datos no presentados).

En la Figura 5 A se observa que no hubo un efecto de los lixiviados sobre la germinación de $V$. sativa en recipientes con sustrato sólido. Tampoco se observó efecto significativo para la mayoría de los lixiviados respecto de la longitud de plántula, detectándose una reducción de longitud de radícula sólo para P. caespitosum, en comparación 
con el testigo y tres de las especies ( $T$. repens, $F$. arundinacea y $S$. halepense) (Figura 5 B). Respecto del efecto sobre la germinación de semillas de $T$. repens, no se verificó inhibición en la germinación (Figura 6 A), ni de longitud de hipocótilo y radícula (Figura 6 B).

En definitiva, no se comprobó el efecto alelopático de los lixiviados en la germinación de una especie sensible como L. sativa, ni hacia otras especies como $T$. repens y $V$. sativa. Tampoco hubo efectos alelopáticos notorios sobre la longitud de hipocótilo y de la radícula. Se presentaron algunos resultados dispares, principalmente al evaluar la longitud de radícula en plántula. En algunos casos se inhibió (L. sativa con lixiviado de S. cryptandrus) y en otros se estimuló ( $L$. sativa con lixiviado de $D$. californica), según la semilla ensayada y el tratamiento de lixiviado.
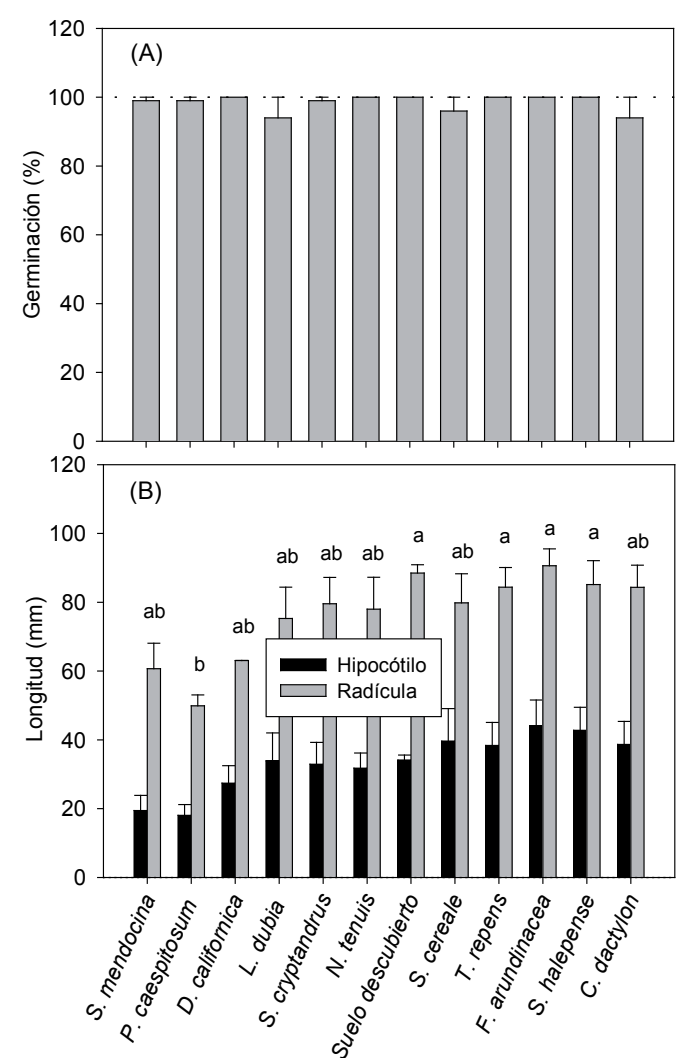

Figura 5. Efecto del lixiviado de diferentes coberturas vegetales en la germinación (A) y en la longitud de plántula (B), discriminada en hipocótilo (barras negras) y radícula (barras grises), de Vicia sativa. Ensayo efectuado en macetas con sustrato. Valores obtenidos siete días después del tratamiento. Octubre de 2009. Letras distintas indican diferencias estadísticas significativas entre las medias (prueba de Tukey; $\rho \leq 0,05 ; n=4$ ). Las líneas verticales muestran el error estándar.
No se detectaron diferencias significativas en la germinación para ninguna de las concentraciones estudiadas (2,5:1, 5:1 y 10:1) ni con el testigo (Figura $7 \mathrm{~A}, \mathrm{~B}$ y $\mathrm{C}$ ), aunque con la mayor concentración se insinúa una tendencia de disminución de la germinación respecto del testigo con agua destilada, pero no respecto del suelo descubierto (Figura 7 C).

En la Figura 8 se analiza el efecto de los concentrados en la longitud de hipocótilo y radícula. Para la concentración 2,5:1 no se apreciaron diferencias entre las especies evaluadas y el suelo descubierto, pero sí una reducción de radícula con relación al testigo con agua destilada (Figura 8 A). En el concentrado 5:1, el tratamiento con lixiviados de $S$. halepense presentó la misma longitud de radícula que el testigo con agua y significativamente mayor a la de suelo descubierto y $N$. tenuis (Figu-
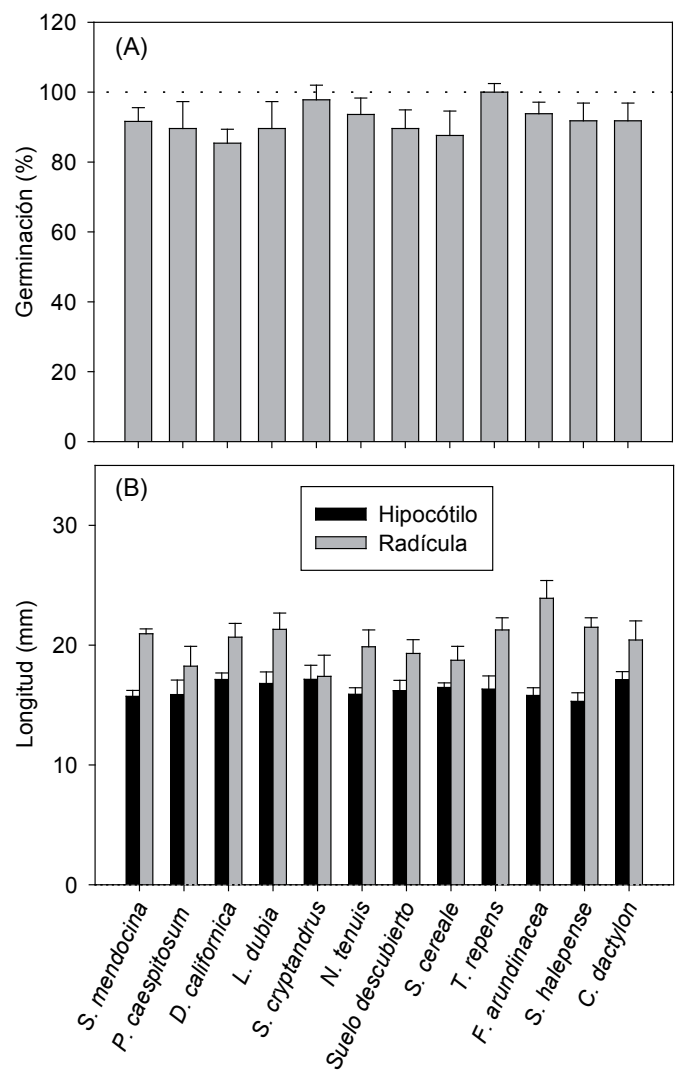

Figura 6. Efecto del lixiviado de diferentes coberturas vegetales en la germinación (A) y en la longitud de plántula (B), discriminada en hipocótilo (barras negras) y radícula (barras grises), de Trifolium repens. Ensayo efectuado en macetas con sustrato. Valores obtenidos siete días después del tratamiento. Octubre de 2009. Sin diferencias estadísticas significativas entre las medias (prueba de Tukey; $\rho \leq 0,05 ; n=5$ ). Las líneas verticales muestran el error estándar. 


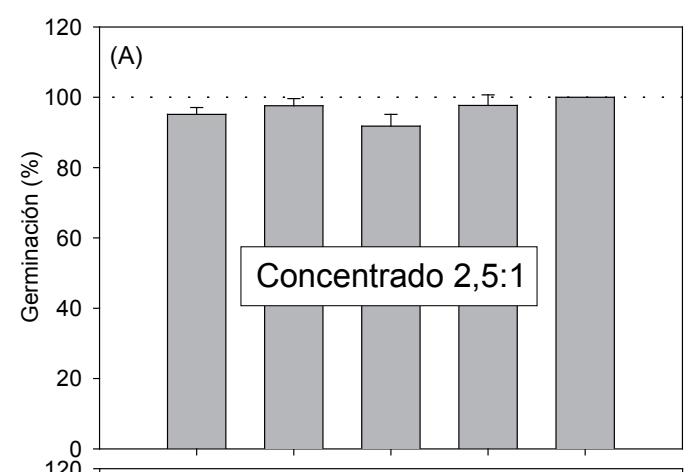

(B)

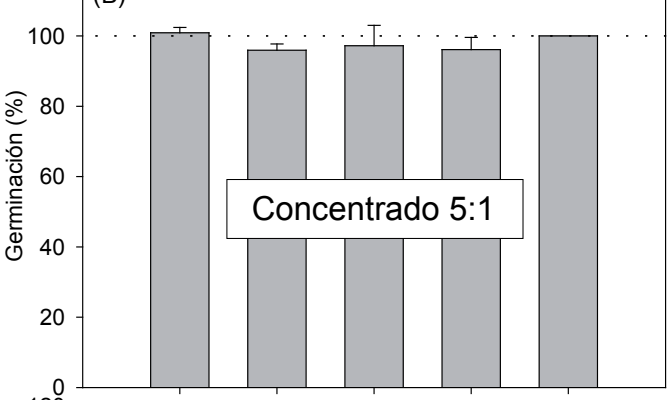

(C)

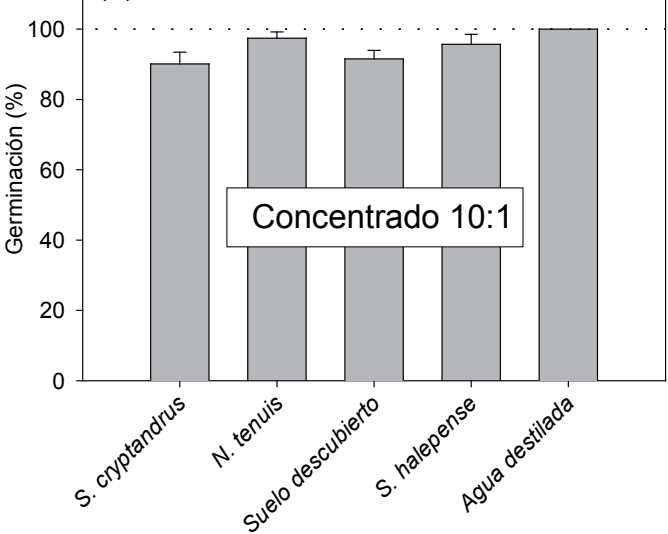

Figura 7. Efecto del lixiviado concentrado de diferentes coberturas vegetales en la germinación de Lactuca sativa cv. Rapidimor clara. (A) Concentrado 2,5:1, (B) concentrado 5:1 y (C) concentrado 10:1. Ensayo efectuado en cámara de germinación. Valores obtenidos cinco días después del tratamiento. Entre mayo y junio de 2009. Sin diferencias estadísticas significativas entre las medias (prueba de Tukey; $\rho \leq 0,05 ; n=5$ ). Las líneas verticales muestran el error estándar.

ra 8 B). Con la concentración 10:1, la longitud de hipocótilo de $S$. halepense fue igual al tratamiento con agua y superior al resto de los tratamientos, mientras que la radícula con lixiviados de $S$. cryptandrus resultó significativamente menor a la del
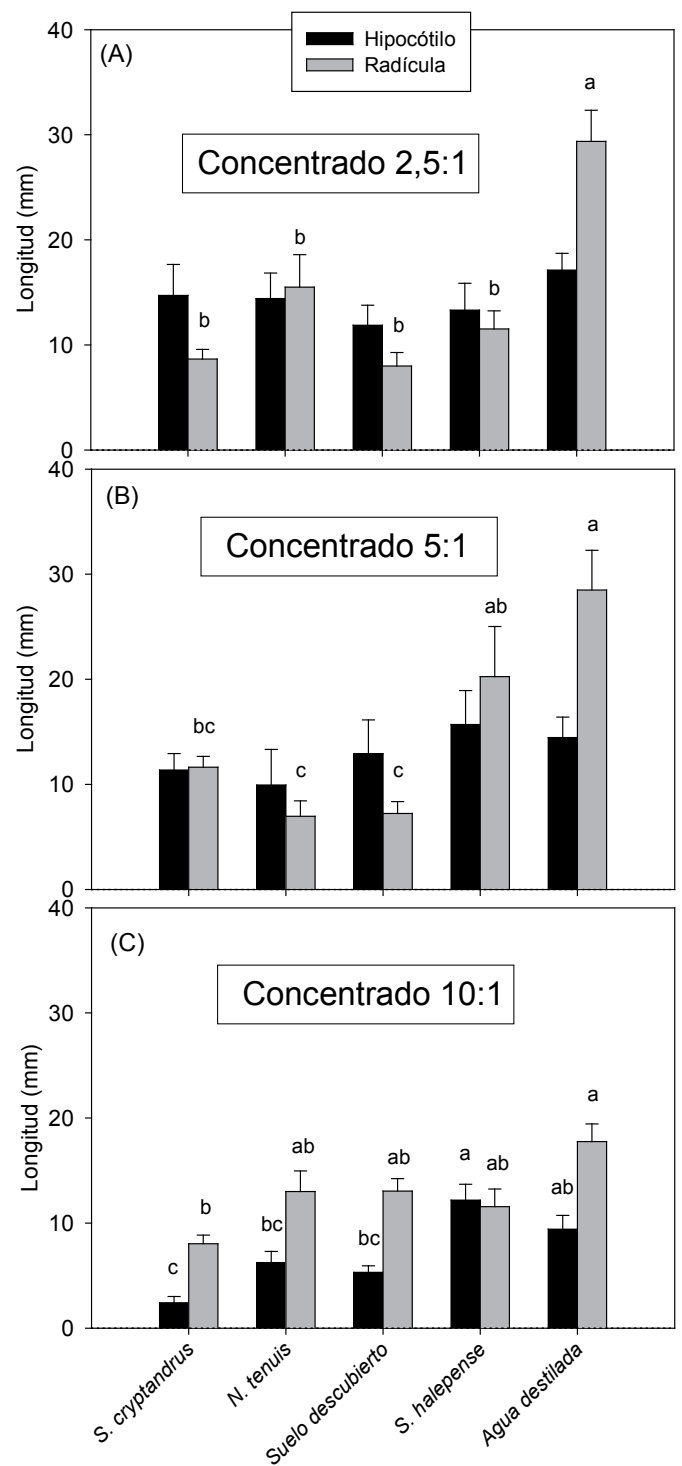

Figura 8. Efecto del lixiviado concentrado de diferentes coberturas vegetales en la longitud de plántula, discriminada en hipocótilo y radícula, de semillas de Lactuca sativa cv. Rapidimor clara. (A) Concentrado 2,5:1, (B) concentrado 5:1 y (C) concentrado 10:1. Ensayo efectuado en cámara de germinación. Valores obtenidos cinco días después del tratamiento. Entre mayo y junio de 2009. Letras distintas indican diferencias estadísticas significativas entre las medias (prueba de Tukey; $\rho \leq 0,05 ; n=5$ ). Las líneas verticales muestran el error estándar

testigo con agua (Figura $8 \mathrm{C}$ ). No se manifestó un efecto inhibitorio de los lixiviados concentrados, cuando se los comparó con el lixiviado del suelo descubierto. Se esperaba que los lixiviados concentrados revelaran algún efecto o tendencia de 
inhibición; sin embargo, no tuvieron efecto sobre la germinación y mostraron resultados dispares sobre la longitud de hipocótilo y radícula.

\section{Contenido de sustancias alelopáticas en los lixiviados}

Los análisis efectuados no evidenciaron la presencia de ninguna sustancia hidrosoluble con actividad alelopática, posiblemente debido a que se trata de un lixiviado de riego de elevada dilución o por efecto de los altos contenidos de materia orgánica y gran capacidad de intercambio catiónico (CIC) del sustrato utilizado. El sustrato de los cultivos de cobertura tuvo una CIC de 34,2 $\mathrm{meq}_{\%} \mathrm{~g}^{-1}$, en comparación con valores de 10,5 $\mathrm{meq} \mathrm{gg}^{-1}$ típicos de un suelo franco de la zona, sin embargo su valor está dentro del rango típico para suelos arcillosos (30 a $35 \mathrm{meq}^{2} \mathrm{~g}^{-1}$ ). Se ha comprobado que la materia orgánica puede alterar el comportamiento de las sustancias alelopáticas debido a fenómenos de sorción (Tharayil et al., 2006).

La información reunida en los diferentes ensayos no determinó ningún efecto alelopático evidente, que justificase profundizar los estudios sobre alguna especie en particular o bien la búsqueda de un compuesto aleloquímico específico en los lixiviados.

Thijs et al. (1994) expresan que previo a la comprensión de estudios químicos exhaustivos como el aislamiento, determinación y cuantificación de aleloquímicos, existe la necesidad de desarrollar métodos experimentales que proporcionen una clara evidencia de los efectos alelopáticos entre las plantas.

La actividad alelopática se ha comprobado fundamentalmente en ensayos de laboratorio, diseñados para maximizar la liberación de aleloquímicos, que de otra manera no se encontrarían en la naturaleza. No muchos de los ensayos de laboratorio prestan atención a los factores edáficos y climáticos, la ecología microbiana, la dinámica de nutrientes, la densidad y otras características bióticas de la planta agresiva (Inderjit \& Weston, 2000), por lo que resulta difícil comprobar su incidencia bajo condiciones de campo. En algunos casos se han encontrado efectos alelopáticos de coberturas verdes sobre porta-injertos de vid, pero utilizando extractos vegetales obtenidos en laboratorio (Bettoni et al., 2012).

Inderjit \& del Moral (1997) afirman que es esencialmente imposible separar alelopatía de competencia por recursos en los sistemas naturales, y que cualquier esfuerzo por separarlos bajo condiciones experimentales creará condiciones que nunca ocurrirían naturalmente.

El hecho de no manifestarse evidencias significativas del efecto alelopático de los lixiviados de diversos cultivos de cobertura, no implica que éstos no interaccionen negativamente con las plantas de vid, a través de la competencia por recursos. Al respecto Müller et al. (1984) observaron que la disminución en el crecimiento de las cepas se correlacionó con una mayor producción de materia seca en las plantas de cobertura, y concluyeron que la interacción más importante fue la competencia por nutrientes, aunque en condiciones de sequía la competencia por agua superó a la competencia por nutrientes.

El presente trabajo sólo consideró el potencial alelopático de sustancias hidrosolubles, exudadas o producto de la descomposición de material vegetal, arrastradas por el agua de riego. Existen otras rutas de acción de los aleloquímicos, como la liberación de sustancias volátiles desde las partes aéreas de las plantas o producto de la descomposición de residuos vegetales y raíces (Anaya, 1999). No obstante ello, las especies de las familias Poaceas (gramíneas) y Fabaceas (leguminosas) no son reconocidas por la producción de dichas sustancias (principalmente terpenos) (Leicach, 2006).

\section{CONCLUSIONES}

Los resultados alcanzados sugieren que, bajo las condiciones de experimentación, no existe un efecto inhibitorio significativo de los lixiviados acuosos estudiados sobre el crecimiento de plantas jóvenes de vid ni tampoco hacia la germinación de otras especies vegetales. Los efectos negativos, tanto de malezas como de coberturas vegetales, que se observan generalmente en el campo, pueden ser atribuidos en mayor medida a la competencia por recursos (agua y nutrientes), sin descartar la intervención de ciertas interacciones alelopáticas. Por lo tanto, al referirse a estas inhibiciones se propone utilizar el término "interferencia", que incluye tanto competencia como alelopatía (Müller, 1966).

El ensayo en macetas mostró que existe una interacción entre las especies y el suelo, mediante la cual el balance de nutrientes es alterado por el tipo de cultivo de cobertura, y que puede afectar positiva o negativamente el crecimiento de las plantas de vid.

Los resultados obtenidos deben ser validados con experiencias de campo para verificar la interacción de los cultivos de cobertura y las plantas 
de vid bajo condiciones naturales.

\section{AGRADECIMIENTOS}

A los doctores A. Tapia y G. Feresin por sus recomendaciones respecto del estudio de sustancias naturales. A M. Fanzone, M. Gomez y J. Torres por el asesoramiento específico en técnicas de laboratorio. A los colegas G. Babelis, J. Maffei y L.E. Martínez, por su colaboración en el análisis de suelos y sustratos. A los compañeros de tareas: V. Rodríguez, F. Ferrari, M. Bonada, M. Montoya, J. Guarise, A. Ambrogetti, A. Porro, P. Díaz y G. Montoya.

\section{BIBLIOGRAFÍA}

Anaya, A.L., 1999. Allelopathy as a tool in the management of biotic resources in agroecosystems. Critical Reviews in Plant Sciences 18(6):697-739.

Bais, H.P., T.L. Weir, L.G. Perry, S. Gilroy and J.M. Vivan$\mathrm{co}, 2006$. The role of root exudates in rhizosphere interactions with plants and other organisms. Annual Review of Plant Biology 57(1):233-266

Bengoa, R., 1983. Efecto alelopático de cinco malezas perennes sobre el crecimiento de plantas jóvenes de duraznero, Universidad de Chile. 116 pp.

Bertin, C., X. Yang and L.A. Weston, 2003. The role of root exudates and allelochemicals in the rhizosphere. Plant and Soil 256(1):67-83.

Bettoni, J.C., J.P.P. Gardin, N.P. Feldberg e M.A. Dalbo, 2012. Potencial alelopático de plantas de cobertura verde de inverno sobre o crescimento do porta-enxerto VR043-43. Revista Ceres 59(1):136-141.

Blum, U., 1996. Allelopathic interactions involving phenolic acids. Journal of Nematology 28(3):259-267.

Bordelon, B.P. and S.C. Weller, 1997. Preplant cover crops affect weed and vine growth in first-year vineyards. HortScience 32(6):1040-1043.

Celette, F., J. Wery, E. Chantelot, J. Celette and C. Gary, 2005. Belowground interactions in a Vine (Vitis vinifera L.)-Tall Fescue (Festuca arundinacea Shreb.) intercropping system: water relations and growth. Plant and Soil 276(1):205-217.

Celette, F., A. Findeling and C. Gary, 2009. Competition for nitrogen in an unfertilized intercropping system: The case of an association of grapevine and grass cover in a Mediterranean climate. European Journal of Agronomy 30(1):41-51.

Cheema, Z.A., A. Khaliq, M. Abbas and M. Farooq, 2007. Allelopathic potential of sorghum (Sorghum bicolor L. Moench) cultivars for weed management. Allelopathy
Journal 20(1):167-178

Chick, T.A. and J.J. Kielbaso, 1998. Allelopathy as an Inhibition factor in ornamental tree growth: implications from the literature. Journal of Arboriculture 24(5):274279.

Di Rienzo, J.A., F. Casanoves, M.G. Balzarini, L. Gonzalez, M. Tablada y C.W. Robledo, 2011. InfoStat. Grupo InfoStat F.C.A. Universidad Nacional de Córdoba, Argentina.

Einhellig, F.A., 1996. Interactions involving allelopathy in cropping systems. Agronomy Journal 88(6):886-893.

Groupe Columa Vigne, 2004. L'enherbement: ANPP. 18 pp.

Inderjit, 2001. Soil: environmental effects on allelochemical activity. Agronomy Journal 93(1):79-84.

Inderjit, 2005. Soil microorganisms: an important determinant of allelopathic activity. Plant and Soil 274(1):227236.

Inderjit and R. del Moral, 1997. Is separating resource competition from allelopathy realistic? The Botanical Review 63(3):221-230.

Inderjit and K.I. Keating, 1999. Allelopathy: principles, procedures, processes, and promises for biological control. Advances in Agronomy 67:141-231.

Inderjit and L.A. Weston, 2000. Are laboratory bioassays for allelopathy suitable for prediction of field responses? Journal of Chemical Ecology 26(9):2111-2118.

Ingels, C.A., R.L. Bugg, G.T. McGourty and L.P. Christensen, 1998. Cover cropping in vineyards, a grower's handbook. Volume Publication 3338. Oakland, California: U.C. A.N.R. 162 pp.

Lambers, H., F.S.I. Chapin and T.L. Pons, 1998. Plant physiological ecology. Berlin Heidelberg New York: Springer. 540 pp.

Leicach, S.R., 2006. Alelopatía. Interacciones químicas en la comunicación y defensa de las plantas. Buenos Aires: Eudeba. 208 pp.

Leicach, S.R., 2009. Biomoléculas. Estructura y rol en el crecimiento y supervivencia de las plantas. Buenos Aires: Editorial Facultad de Agronomía. Universidad de Buenos Aires. 305 pp.

Macías, F.A., J.M.G. Molinillo, R.M. Varela and J.C.G. Galindo, 2007. Allelopathy - a natural alternative for weed control. Pest Management Science 63(4):327-348.

Marschner, H., V. Römheld and I. Cakmak, 1987. Rootinduced changes of nutrient availability in the rhizosphere. Journal of Plant Nutrition 10(9-16):1175-1184.

Monteiro, A. and C.M. Lopes, 2007. Influence of cover crop on water use and performance of vineyard in Mediterranean Portugal. Agriculture, Ecosystems \& Environment 121(4):336-342.

Müller, C.H., 1966. The role of chemical inhibition (allelo- 
pathy) in vegetational composition. Bulletin of the Torrey Botanical Club 93(5):332-351.

Müller, W., E. Rühl und H. Gebbing, 1984. Untersuchungen über die wechselwirkung zwischen rebe und begrünungspflanzen. Die Wein-Wissenschaft (39):3-15.

Ohno, T. and K.L. Doolan, 2001. Effects of red clover decomposition on phytotoxicity to wild mustard seedling growth. Applied Soil Ecology 16(2):187-192.

Paynel, F., P. J Murray and J. Bernard Cliquet, 2001. Root exudates: a pathway for short-term $\mathrm{N}$ transfer from clover and ryegrass. Plant and Soil 229(2):235-243.

Peters, E.J. and K.T. Luu, 1985 Allelopathy in Tall Fescue. In: The Chemistry of Allelopathy. ACS Symposium Series, Vol. 268: American Chemical Society. pp. 273283.

Römer, W. and G. Schilling, 1986. Phosphorus requirements of the wheat plant in various stages of its life cycle. Plant and Soil 91(2):221-229.

Sampietro, D.A., 2007. Alelopatía: concepto, características, metodología de estudio e importancia. Universidad Nacional de Tucumán, San Miguel de Tucumán. $26 \mathrm{pp}$.

Tharayil, N., P.C. Bhowmik and B. Xing, 2006. Preferential sorption of phenolic phytotoxins to soil: Implications for altering the availability of allelochemicals. Journal of Agricultural and Food Chemistry 54(8):3033-3040.

Thijs, H., J.R. Shann and J.D. Weidenhamer, 1994. The effect of phytotoxins on competitive outcome in a model system. Ecology 75(7):1959-1964.

Uliarte, E.M., H.R. Schultz, C. Frings, M. Pfister, C.A. Parera and R.F. del Monte, 2013. Seasonal dynamics of $\mathrm{CO}_{2}$ balance and water consumption of $\mathrm{C}_{3}$ and $\mathrm{C}_{4}$-type cover crops compared to bare soil in a suitability study for their use in vineyards in Germany and Argentina. Agricultural and Forest Meteorology 181:1-16.

Uliarte, E.M., C.A. Parera, E.E. Alessandria y A.D. Dalmasso, 2014. Intercambio gaseoso y eficiencia en el uso del agua de cultivos de cobertura con especies nativas (Mendoza, Argentina), exóticas cultivadas y malezas. AgriScientia 31(2):49-61.

Velu, G. and A. Rajagopal, 1996. Allelopathic impact of purple nutsedge (Cyperus rotundus) and bermudagrass (Cynodon dactylon) on soybean (Glycine max). Indian J. Agric. Sci. 66:363-365.

Whitelaw, M.A., 2000. Growth promotion of plants inoculated with phosphate solubilizing fungi. Advances in Agronomy 69:99-151.. 